60-61| 2018

La culture dans l'enseignement du français langue étrangère: conceptions théoriques, programmes et manuels auX $\mathrm{XIX}^{\mathrm{e}}$ et $\mathrm{XX}^{\mathrm{e}}$ siècles

\title{
Les langues vivantes à l'école russe après la réforme de 1871 : recherche de revanche ou défi de modernisation?
}

Modern languages in Russian schools after the 1871 reform: a quest for revenge or a challenge to modernise?

Nadéjda Kriajeva

\section{OpenEdition}

Édition électronique

URL : https://journals.openedition.org/dhfles/5747

DOI : $10.4000 /$ dhfles. 5747

ISSN : 2221-4038

Éditeur

Société Internationale pour l'Histoire du Français Langue Étrangère ou Seconde

Édition imprimée

Date de publication : 1 janvier 2018

Pagination : $361-379$

ISSN : 0992-7654

Référence électronique

Nadéjda Kriajeva, « Les langues vivantes à l'école russe après la réforme de 1871 : recherche de revanche ou défi de modernisation ? ", Documents pour l'histoire du français langue étrangère ou seconde [En ligne], 60-61 | 2018, mis en ligne le 01 juin 2019, consulté le 29 mars 2023. URL : http:// journals.openedition.org/dhfles/5747 ; DOI : https://doi.org/10.4000/dhfles.5747

Ce document a été généré automatiquement le 29 mars 2023.

Tous droits réservés 


\title{
Les langues vivantes à l'école russe après la réforme de 1871 : recherche de revanche ou défi de modernisation?
}

\author{
Modern languages in Russian schools after the 1871 reform: a quest for revenge \\ or a challenge to modernise?
}

Nadéjda Kriajeva

\section{Vers un nouvel état des lieux}

1 Dès l'affirmation officielle du système éducatif russe possédant tous les niveaux (élémentaire, secondaire et supérieur) en 1803, le contenu de l'enseignement a fait l'objet des préoccupations principales des milieux pédagogiques, et les réformes menées par le ministère de l'Instruction publique lui donnent des configurations différentes à plusieurs reprises. Les langues principales enseignées à l'école secondaire, le russe et le slavon d'église, les langues étrangères vivantes (LV), le français, l'allemand, l'anglais et enfin les langues anciennes (le latin et le grec) qui l'ont intégrée en dernier, se trouvent au centre des débats pédagogiques d'une rare vivacité et d'une grande ampleur durant toute la seconde moitié du XIX et le début du XX ${ }^{\mathrm{e}}$ siècle.

L'année 1871 marque le début d'une nouvelle étape qui sera la dernière dans l'histoire de l'école secondaire de la Russie impériale. Cette date est aussi importante pour le même domaine en France, quoique pour des raisons au premier abord différentes mais en réalité très proches : il s'agissait de la rénovation des contenus de l'enseignement secondaire et de la place que les langues anciennes devaient occuper par rapport aux modernes. En effet, en 1871, l'école secondaire russe qui existait depuis le début du XIX ${ }^{e}$ siècle, a connu une réforme marquante des cursus. Avec cette réforme, les élèves s'engageaient soit dans une filière dite moderne soit dans une filière dite classique 
selon le type d'établissement choisi et le sexe. Cette réforme en faveur du classicisme très strict des gymnases est dite "tolstovienne" parce que mise en place sous le ministère (1866-1880) du comte Dimitri Tolstoï (1823-1889). Soigneusement préparée, longuement débattue à tous les niveaux pendant six ans, elle a mis fin aux discussions des pédagogues des deux partis opposés et aux fluctuations dans le système éducatif des années précédentes et a valu une réputation controversée à son initiateur.

Plusieurs historiens contemporains spécialistes de la Russie, comme beaucoup d'historiens russes et soviétiques du domaine de l'instruction publique (Kornilov 1914, Ganelin 1950), continuent d'y voir une décision politique et rétrograde :

« L'ère Tolstoï » fut, pour l'université et pour l'éducation en général, l'une des plus néfastes de l'histoire russe. Les plus redoutables décisions de Tolstoï pour l'avenir intellectuel de toute une génération touchaient à sa conception du contenu souhaitable des études. Il imputait au progrès des connaissances scientifiques, que les réformes de son prédécesseur avaient assuré, le développement de l'esprit «matérialiste et superficiel » de la jeunesse. [...] En revanche, l'accent fut mis sur les langues mortes, la grammaire et les mathématiques, jugées peu dangereuses. (Carrère d'Encausse 2008 : 267-268).

4 En effet depuis la réforme, les universités ne sont plus ouvertes qu'aux élèves des gymnases de garçons, exigeant l'étude de deux langues anciennes (le latin et le grec). Elles ne sont plus accessibles aux sortants d'établissements de type moderne : anciens gymnases communs et réaux transformés en écoles réales ${ }^{1}$. Ces établissements de type moderne n'ouvrent la voie des hautes études que partiellement, à savoir aux écoles supérieures spécialisées ${ }^{2}$ et non aux études en lettres, histoire, philosophie, droit. Certes, on peut y voir un durcissement politique si l'on se focalise sur le fait que la réforme avait été imposée par la volonté du ministre; pour la faire adopter, Tolstoï s'appuyait sur une petite minorité du Conseil d'État (dernière instance de validation des réformes) et sur un vif soutien de l'empereur Alexandre II. On oublie pourtant (volontairement ou non ${ }^{3}$ ) que Tolstoï, tout en favorisant les gymnases classiques, ne délaissa pas le développement du contenu moderne de l'enseignement secondaire. Or, dans la société, l'attitude par rapport à la réforme n'avait pas été aussi négative (Stépanov 1907) que l'ont prétendu les historiens engagés idéologiquement durant tout le XX $\mathrm{XX}^{\mathrm{e}}$ siècle. L'intérêt pour ces enjeux et pour ces leçons dans la Russie postsoviétique est spectaculaire, car c'est sous-jacent à la recherche de contenus éducatifs nouveaux et diversifiés. Dans ce contexte nettement réformiste, les questions de réintégration des langues anciennes et le rôle des langues tout court à l'école actuelle refont surface ${ }^{4}$. Cela est favorisé par un accès libre de toutes contraintes aux différents fonds d'archives, aux revues pédagogiques d'antan ${ }^{5}$ et aux collections d'outils didactiques, tombés dans l'oubli auparavant ${ }^{6}$. En explorant ce nouveau contexte, nous avons été confrontée néanmoins à l'absence de description du corpus des livres scolaires utilisés pour l'enseignement des LV dans la Russie impériale. Nombreux et d'une grande diversité, ces livres sont éparpillés dans les bibliothèques du pays et souvent ignorés ou mésestimés des historiens de la didactique des $\mathrm{LV}^{7}$. Une plongée dans des sources primaires de la période en question nous a permis de comprendre, bien différemment sur plusieurs points, certains changements apportés par la réforme par rapport à leurs évaluations existantes, ce que nous allons démontrer dans cet article. 


\section{Diversification des objectifs et des plans d'études}

5 La réforme tolstovienne différencie les objectifs d'enseignement des LV pour les établissements classiques réservés aux garçons et les établissements de type moderne. Les différences établies par les plans d'études de 1872 se mettent progressivement en place avec des modifications de détail selon les directives ministérielles (1890-1892, $1895,1902,1905)$ qui portent sur le nombre d'heures, sur les conditions de cours, etc. Par exemple, la note explicative au programme des LV dans les gymnases de garçons (1890) formule les objectifs en ces termes:

procurer à la jeunesse une éducation générale, et en même temps préparer à l'entrée à l'Université et dans les hautes écoles spéciales, l'enseignement des LV poursuit un double but : concourir à l'éducation générale des élèves en développant leurs facultés intellectuelles, et leur donner les notions indispensables pour qu'à la sortie du gymnase ils soient en état de lire les ouvrages scientifiques et littéraires allemands et français, tant en vue d'élargir leur éducation générale que d'étudier un sujet spécial de leur choix 8 .

6 Cette notice a l'empreinte de l'approche pratique, consciente et contrastive, théorisée en Russie dans les années 1860 par Fedor Buslaev (1818-1897) initialement pour la langue maternelle (Kriajeva 2017). Ayant eu un vif succès à l'école, cette approche est élargie aux langues étrangères. Pour la résumer: la maîtrise consciente de la grammaire et du vocabulaire de la langue maternelle crée (du point de vue linguistique et psychologique) des fondements très solides pour l'étude de toute autre langue. Elle en est une condition préalable. Cette théorie, mise en pratique officiellement par la réforme classique de 1872, réunit les idiomes étudiés à l'école en un ensemble hiérarchisé selon l'importance de chacun d'eux dans la construction du «savoir linguistique conscient et approfondi » (ibid.). Dans cet ensemble, la langue russe et le slavon d'église sont secondés par le latin et le grec. Les langues modernes (le français et l'allemand) l'intègrent un peu plus tard car leur potentiel éducatif est considéré comme moins formateur, ayant elles mêmes pour base les langues anciennes. Cette dernière opinion qui avait cours depuis la création des premiers gymnases classiques (1828) commence, vers les années 1860 , à sérieusement contester la suprématie du français et de l'allemand, solidement établis dans le système éducatif russe depuis la seconde moitié du XVIII e siècle (Stepanov 1907, Alešincev 1912, Kouzmina 2005). Les enseignants devaient désormais démontrer et défendre l'importance des LV pour l'instruction secondaire, comme le fit par exemple David Margot $^{9}$ en 1864 :

Le travail d'observation et d'analyse doit former le point de départ dans l'étude des langues, c'est là un des moyens les plus efficaces de cultiver les jeunes intelligences [...]. C'est pour avoir méconnu cette vérité, et confondu la grammaire avec la routine, que des hommes, induits en erreur sans doute par une expérience incomplète, ont porté sur l'enseignement des langues modernes un jugement qu'un maître, éclairé par une longue expérience et l'amour de sa vocation, ne peut que repousser de toutes ses forces. [...] Des esprits graves reprochent aux langues modernes, et au français en particulier, de n'être pas, pour les élèves des classes supérieures, un travail aussi efficace que celui que procure l'étude du latin et du grec.

Sans vouloir contester aux langues anciennes leur supériorité au point de vue qui nous occupe, il est évident que le reproche fait à l'enseignement des langues modernes doit s'adresser à la position faite à cet enseignement dans les écoles bien plus qu'à la langue elle-même, qui, comme l'expérience le démontre, offre un moyen d'éducation des plus précieux (Margot 1896 [1864] : III-IV). 
7 On trouve le même type de discours chez d'autres enseignants de français, auteurs de grammaires et manuels réputés comme Varon (1874: 90-91) ou Fleury (1872: III). Plus tard, on souligne toujours que " les langues modernes sont aussi civilisatrices et aussi plastiques que le latin et le grec » et en plus les LV donnent « l'accès au progrès et aux cultures du monde contemporain » (Kerkoff $1892: \mathrm{X}$ ).

8 L'idée de complémentarité dans l'apprentissage des langues, incarnée dans l'approche consciente et contrastive, réconciliait en quelque sorte les enseignants de toutes les langues ${ }^{10}$. Pourtant, la réticence vis-à-vis de l'expression orale en LV en tant qu'objectif pour les gymnases classiques était évidente. Cette compétence n'y est pas érigée en but à atteindre: savoir simplement parler une langue moderne ne signifierait pas sa véritable connaissance qui demeure plutôt dans ses meilleures œuvres littéraires. Cela se ralliait à l'opinion très répandue sur la pratique du français au quotidien entre les gens nobles et cultivés, vue toujours comme "une sorte de nécessité ${ }^{11}$. Par conséquent, l'acquisition d'un tel usage devait se faire en dehors de l'école. Les programmes des gymnases classiques ne mettaient pas en avant la formation de compétences en expression orale pour les LV; il y avait même de la méfiance à son encontre : « causer » en français ou en allemand serait trop primitif pour les gymnases, considéré comme une perte de temps précieux ${ }^{12}$.

9 Konstantin Ušinskij (1824 -1870), pédagogue et partisan de l'approche consciente des langues l'affirme clairement en polémiquant à ce sujet: "les moyens utilisés dans l'apprentissage des langues sont prédéfinis par les objectifs qu'on se donne ». Il en distingue quatre: 1) pouvoir accéder aux trésors culturels (littérature) de la langue apprise ; 2) concourir au développement intellectuel des élèves ; 3) communiquer avec les habitants de pays étrangers dans leurs langues natales ; 4) communiquer avec ses compatriotes qui ont appris la même langue étrangère. Ušinskij ridiculise le dernier objectif, visant en particulier l'usage du français dans les milieux nobles : «Il est hors de doute que l'importance du premier but offrant les clés de la culture d'un autre peuple s'avère autant incontestable que l'est la futilité du dernier qui nous fait suivre une mode étrange et perverse : parler avec ses propres compatriotes dans une langue étrangère" (Ušinskij 1861: 87). Selon lui, seuls les deux premiers méritent d'être honorés dans les gymnases: la littérature dans une langue originelle vivante « donne les clés de la culture d'un peuple» et savoir analyser de nouvelles structures linguistiques renforce l'intelligence.

10 C'est cette approche qui sera privilégiée dans les gymnases classiques qui évolueront moins vite vers la méthodologie directe (et mixte) que les écoles de type moderne. La difficulté de son application aux LV dans les gymnases se manifeste de plus en plus vers la fin $\mathrm{du} \mathrm{XIX}^{\mathrm{e}}$ siècle: un minimum de compétences langagières en LV acquis habituellement au préalable (précepteurs en famille) n'était plus au rendez-vous, le public d'élèves s'étant élargi et démocratisé. Mais l'entente sur ce terrain sera trouvée un peu plus tard, vers la fin du XIX ${ }^{e}$ siècle, sous l'impact promotionnel de la méthodologie directe et cela grâce aux efforts de ses partisans qui exerçaient en principe dans les deux types d'écoles.

11 Pour les établissements de type moderne (avec deux, voire trois LV) les objectifs ont été formulés différemment avec une insistance sur le côté «pratique». Ainsi pour les établissements de filles :

À la fin des études au gymnase, les jeunes filles doivent être capables de comprendre des œuvres du genre historique ou narratif ne présentant pas de trop 
grandes difficultés. Il est aussi souhaitable qu'elles s'expriment avec aisance au moins dans l'une des langues apprises, dans la mesure où la plupart d'entre elles se destinent à l'exercice du métier de gouvernantes dans les familles. Il est nécessaire que leur soit assurée la possibilité de communiquer en langues étrangères au sein de l'établissement avec le personnel encadrant (surveillantes, dames de classe, directrice) en dehors des cours. Dans les classes supérieures (VI et VII), les littératures étrangères seront enseignées dans les langues apprises (auteurs, œuvres, périodes et tendances littéraires), les demoiselles devront s'exercer en rédaction de lettres et d'exposés concernant les sujets appris et/ou la vie quotidienne $^{13 .}$

Pour les écoles réales :

Les garçons doivent entendre les textes non compliqués traitant de sujets historiques, narratifs et techniques, savoir mener la correspondance écrite élémentaire concernant ces domaines ainsi que la vie quotidienne [...]. Après une année supplémentaire (en $7^{\mathrm{e}}$ ), les élèves doivent savoir traduire des articles (du russe vers les LV) plus difficiles portant sur des sujets généraux, techniques et/ou commerciaux; savoir s'exprimer (oralement et par écrit) et mener une conversation dans les langues étudiées $[. . .]^{14}$.

13 Le caractère pratique de l'apprentissage du français, de l'allemand et plus tard de l'anglais, est accentué en termes similaires dans les plans d'études de toutes les écoles de type moderne : «s'exprimer avec aisance », savoir " mener la correspondance écrite en général et dans leur spécialité », pouvoir " relater les œuvres prosaïques faciles ", etc. Mais entre 1898 et 1903, un compromis entre les deux approches est trouvé, les idées directrices des plans d'études pour les LV dans les établissements des deux types deviennent communes, scellant en même temps l'alliance des méthodes traditionnelles et directes :

Dans les petites classes, on doit se servir de la méthode naturelle ou américaine pour que les élèves apprennent à s'exprimer en langues étrangères oralement et par écrit ; la conversation orale constante en langues étrangères est le lien qui unira toutes les classes, et sous ce nom de conversation, on comprendra la conversation orale, la paraphrase et les reproductions des récits par écrit. [...] Les deux leçons hebdomadaires doivent être réservées pour exercer les élèves en écriture: correspondance en langues étudiées, etc. (Nedačin $1904: 160)^{15}$

14 Ce passage du plan d'études d'un gymnase moscovite mixte montre le but nettement prioritaire : savoir s'exprimer et converser dans les langues apprises dans les petites classes de toutes les écoles, y compris les gymnases classiques.

\section{Une toile de fond commune}

15 Les programmes des gymnases pour les classes moyennes et supérieures mettent en avant la dimension culturelle : l'initiation à la lecture des meilleurs auteurs français et allemands doit «favoriser non seulement l'étude pratique de la langue, mais aussi le développement intellectuel de l'élève [...] permettant de faire connaissance avec les auteurs les plus estimés » (Jaclard-Corvin 1878 : I-II). Dès 1890, les cours d'histoire de la littérature française et/ou allemande deviennent obligatoires dans les classes supérieures (de la $5^{\mathrm{e}}$ à la $7^{\mathrm{e}} / 8^{\mathrm{e}}$ classe : deux heures par semaine en plus des heures de chaque LV).

Pour le français, ces cours comprennent les périodes dans l'ordre suivant: en $5^{\mathrm{e}}$ et $6^{\mathrm{e}}$ classes, ce sont les grands prosateurs du XIX siècle et une brève histoire du français 
(langue d'oc et langue d'oïl), les troubadours et les trouvères, Le Roman de Renart et Le roman de la Rose, le romantisme, le réalisme, etc. En $7^{\mathrm{e}}$, ce sont les auteurs dramatiques des $\mathrm{XVII}^{e}, \mathrm{XVIII}^{\mathrm{e}}$ et XIX ${ }^{\mathrm{e}}$ siècle, notamment Corneille, Racine, Molière, Voltaire, Hugo, Musset, etc. et l'initiation à l'analyse d'œuvres littéraires : l'auteur, son époque, les idées directrices de l'œuvre. Le cours de littérature en $7^{\mathrm{e}}$ doit porter sur l'évolution du théâtre en France du Moyen Âge au XIX siècle : la tragédie chez les Anciens et chez les Modernes, le théâtre avant Corneille ; Corneille, Racine, Molière, Voltaire, etc. Le plan de l'étude d'un auteur prévoit sa courte biographie, l'analyse et l'appréciation des œuvres principales. En $8^{\mathrm{e}}$, le cours de littérature porte sur l'évolution du roman (Lesage, l'abbé Prévost, Mme de Staël, Chateaubriand, Vigny, Hugo, Mérimée, Balzac, George Sand, Dumas, Flaubert) et sur l'évolution de la poésie (Marot, Ronsard, Malherbes, La Fontaine, Boileau, Lamartine). Les élèves doivent savoir parler des auteurs étudiés d'après le plan élaboré en $7^{\mathrm{e}}$ et effectuer des compositions et narrations écrites. Les leçons de français dans ces classes sont consacrées en principe à la lecture de fragments littéraires et aux différents exercices permettant leur meilleure compréhension et l'enrichissement du vocabulaire (Nedačin 1904).

Pavel Mižuev (1861-1932), historien et pédagogue, enseignant de français et d'anglais, l'un des acteurs de la modernisation du système éducatif, précisait que «l'étude des LV permet de rendre les cultures étrangères accessibles, elle enrichit l'intelligence par des notions sur la mentalité d'autres peuples, ayant ainsi une valeur inestimable pour la culture générale de la jeunesse » $(1890: 83)$. Dans tout type d'établissement (gymnases, écoles réales ou militaires), l'enseignement des LV dans les classes moyennes et supérieures s'appuie sur des "morceaux littéraires tirés des meilleurs auteurs ». Ils se trouvent intégrés dans des « cours pratiques de langue » (Moser 1897, Challandes 1902), ou figurent dans des chrestomathies élaborées en vue de l'usage scolaire (Feuillye \& Martin 1914, Mižuev 1894, 1911). Pour pouvoir arriver enfin à cette synthèse, il a fallu plusieurs années de débats à propos des modifications de la méthodologie grammaire/ traduction, et son alliance avec la méthodologie directe. Eugène Varon (?-1886), professeur de français qui enseignait dans les gymnases, les écoles réales et aussi à l'Institut pédagogique de St-Pétersbourg, réfléchissant sur la possibilité d'une telle jonction dès 1874 , la voyait vitale et prometteuse. Il était persuadé qu'une «fusion des méthodes directes et grammaticales ", malgré la difficulté d'une telle entreprise, constituait l'avenir de l'apprentissage des LV à l'école (Varon $1874: 58-95)^{16}$. Trentecinq ans après, en 1909, Lanzeff, enseignant de français dans un gymnase de province, l'a indirectement confirmé :

Maintenant que la nouvelle méthode d'enseignement (dite américaine) est adoptée à peu près partout, les élèves possèdent, dès les classes inférieures, un vocabulaire suffisant pour comprendre, sans qu'il soit besoin de traduire, les règles qui sont exposées ici de la manière la plus simple possible. (Lanzeff $1909: 3-4$ )

Cependant, l'étude de la grammaire, sous l'impact de la méthodologie directe, n'est pas abandonnée pour autant mais se fait différemment. On insiste souvent sur ces différences écrivant que dans les classes élémentaires les notions grammaticales seront " ou tout à fait absentes, ou données occasionnellement ». Seulement dès les classes moyennes, les recueils de textes étudiés seront accompagnés d'un exposé allégé de notions grammaticales formant une partie spéciale du manuel. L'essentiel du temps en cours sera consacré au travail autour des textes tirés de chrestomathies car «la véritable grammaire est dans les écrits des bons auteurs" (Challandes 1902: III). Des grammaires "allégées », " dépourvues de détails inutiles et encombrants", adaptées 
aux manuels (Martin 1904, Fabriciev 1903) obtiennent rapidement des appréciations flatteuses dans la presse pédagogique.

\section{Les outils didactiques : spécification et concurrence}

19 Le ministère de l'Instruction publique n'a plus le monopole de l'édition des manuels, mais il est censé encourager la spécification et une libre concurrence dans ce domaine par l'établissement de primes et de concours. Son Comité scientifique peut donner des avis diversifiés sur les ouvrages: "apprécier », "recommander», ou simplement « autoriser à l'usage dans... ». De ce fait, les concepteurs de manuels de LV cherchent à obtenir ces approbations, et aussi celles d'autres comités scientifiques et pédagogiques, pour plus de crédibilité auprès des établissements éducatifs qui sont tous libres dans leurs choix d'outils didactiques.

En observant les livres scolaires de cette période, nous constatons un effort considérable d'adaptation des "cours traditionnels à objectifs pratiques" déjà existants sous la pression de plus en plus évidente de la méthodologie directe qui s'installait activement à l'école depuis la fin du XIX ${ }^{e}$ siècle.

21 Les manuels «concentriques », "gradués », " progressifs », " avec les textes suivis » sont régulièrement complétés par des indications comme : "spécialement adapté à la méthode directe ", « adapté à la progression de l'enseignement par l'image » (Fabriciev 1903, Fenoult 1906, Ihnatovicz 1899, Lanzeff 1909, Moser 1892, Varon 1878). Ils sont en usage à partir des classes moyennes dans les gymnases classiques et à partir des classes supérieures dans d'autres établissements. Il y a par ailleurs une progression rapide des méthodes rédigées (ou adaptées à l'usage des russophones) dans l'esprit de la méthodologie directe : "Berlitz », " américaine », « intuitive », " naturelle » (Feuillye \& Martin 1904, Kerkoff 1892, Klasse 1908, Konof 1904, 1914, Silina 1895, Trilling 1903) ${ }^{17}$. Elles sont utilisées dans les classes inférieures des gymnases, inférieures et moyennes dans les établissements non classiques.

Le contenu culturel, lui, s'avère commun pour les établissements secondaires de tout type. La différence se manifeste par la diversité et la profondeur. Par contenu il faut entendre la connaissance des meilleurs œuvres de littérature étrangère. Les chrestomathies et anthologies de littérature française, rédigées spécialement à l'usage des écoles russes (Jaclard-Corvin 1878, Feuillye \& Martin 1914, Martin 1917) ou adaptées à l'usage des russophones (Fleury 1874), entre les années 1870 et le début du $\mathrm{XX}^{\mathrm{e}}$ siècle sont largement présentes dans l'enseignement à l'école, notamment pour les classes moyennes et supérieures. Elles se divisent en principe en deux catégories. La première est adaptée à l'âge des élèves et à la progression dans l'étude (Margot 1905, Mižuev 1894, 1911, Moser 1892, Konof 1914). La seconde s'organise plutôt selon les principes chronologiques et stylistiques (Feuillye \& Martin 1914, Fleury 1874, JaclardCorvin 1878, Pachalery 1905). Les auteurs pratiquant les deux méthodologies différentes expliquent l'utilité de leurs recueils en termes similaires : «favoriser non seulement l'étude pratique de la langue mais aussi le développement intellectuel de l'élève ", en précisant également qu' "en Russie, comme dans les autres pays, il est beaucoup d'établissements [...] où, par suite de l'importance toujours croissante accordée aux sciences positives ou aux études classiques, [...] il devient indispensable que le manuel en usage pour les lectures » renferme les informations qui permettent de 
«faire connaissance avec les auteurs les plus estimés» (Jaclard-Corvin 1878: I) en initiant ainsi l'élève à la connaissance d'autres peuples et cultures.

À la fin du XIX ${ }^{\mathrm{e}}$ siècle et au début du XXe siècle, la méthodologie directe se fait une belle place en Russie au sein de l'approche "pratique consciente et contrastive» qui l'intègre en tant que partie fondamentale de l'enseignement-apprentissage des LV dans les petites et moyennes classes de l'école secondaire (de tout type). L'effort tangible de rénovation, venant des représentants de la méthodologie traditionnelle, contribue également à l'émergence d'une des configurations de la méthodologie mixte de l'enseignement-apprentissage des LV à l'échelle nationale. Le rôle et le prestige des LV vis-à-vis des langues anciennes et d'autres matières enseignées à l'école secondaire de cette période s'en trouvent donc renforcés. Les LV sont reconnues comme instruments efficaces de l'éducation servant autant au développement intellectuel que culturel des élèves : le défi de modernisation a été relevé.

\section{BIBLIOGRAPHIE}

Sources primaires

Documents et circulaires du ministère de l'Instruction publique de Russie

УЧЕБНЫЙ ПЛАН НОВЫХ ИНОСТРАННЫХ ЯЗЫКОВ (1874). [Plan d'études des langues étrangères vivantes]. ЖУРНАЛ МИНИСТЕРСТВА НАРОДНОГО ПРОСВЕЩЕНИЯ (ŽMNP), Partie 175 : 150- 152.

УЧЕБНЫЕ ПЛАНЫ НОВЫХ ИНОСТРАННЫХ ЯЗЫКОВ ДЛЯ ЖЕНСКИХ СРЕДНЕ-УЧЕБНЫХ ЗАВЕДЕНИЙ (1874).

[Plans d'études des langues vivantes dans les établissements secondaires de filles]. SaintPétersbourg : MNP.

УЧЕБНЫЕ ПЛАНЫ И ПРИМЕРНЫЕ ПРОГРАММЫ УЧЕБНЫХ ПРЕДМЕТОВ, ПРЕПОДАВАЕМЫХ В МУЖСКИХ ГИМНАЗИЯХ И ПРОГИМНАЗИЯХ (1890). [Plans et programmes d'études des gymnases et progymnases de garçons]. Saint-Pétersbourg : MNP.

DUDYŠKIN, Sergej (dir.) (1898). ПРОГРАММЫ РЕАЛЬНЫХ УЧИЛИЩ. СПРАВОЧНИК, [Plans d'études des écoles réales. À l'usage des parents d'élèves]. Moscou : E. Guerbec.

УЧЕБНЫЕ ПЛАНЫ И ПРОГРАММЫ ПРЕДМЕТОВ, ПРЕПОДАВАЕМЫХ В РЕАЛЬНЫХ УЧИЛИЩАХ (1879). [Plans d'études et programmes des matières enseignées dans les écoles réales]. Saint-Pétersbourg: MNP.

NEDAČIN, Vasilij (dir.) (1904). МОСКОВСКАЯ ГИМНАЗИЯ ИМЕНИ МЕДВЕДНИКОВЫХ. ОСНОВЫ УЧЕБНОВОСПИТАТЕЛЬНОЙ ОРГАНИЗАЦИИ. 1901-1904, [Principes d'enseignement et d'éducation dans le gymnase Medvednikov. Moscou : G.Lissner \& A.Gešel.

Ouvrages et articles critiques publiés entre 1861 et 1917

ALEŠINCEV, IVan (1912). ИСТОРИЯ ГИМНАЗИЧЕСКОГО ОБРАЗОВАНИЯ В РОССИИ. ХVIII И ХІХ ВВ., [Histoire de l'instruction dans les gymnases russes au XIX et au XX ${ }^{\mathrm{e}}$ siècles]. Saint-Pétersbourg: 0. Bogdanova. 
BARSKOv, Jakov (dir.) (1914). ПЕДАГОГИЧЕСКИЙ МУЗЕЙ ВОЕННО-УЧЕБНЫХ ЗАВЕДЕНИЙ. 1864-1914. ИСТОРИЧЕСКИЙ ОЧЕРК, [Musée pédagogique des établissements secondaires militaires. Essai d'histoire]. Saint-Pétersbourg, XXVII, 346 c.

MižUEV, Pavel (1890). « ЦЕЛЬ ПРЕПОДАВАНИЯ НОВЫХ ИНОСТРАННЫХ ЯЗЫКОВ В СРЕДНЕ-УЧЕБНЫХ ЗАВЕДЕНИЯХ », [L'objectif de l'enseignement des langues vivantes dans les établissements secondaires]. Russkaja škola, $\mathrm{n}^{\circ} 3:$ 78-90.

STEPANOV, Sergej (1907). ОБОЗРЕНИЕ ПРОЕКТОВ РЕФОРМЫ СРЕДНЕЙ ШКОЛЫ В РОССИИ ПРЕИМУЩЕСТВЕННО В ПОСЛЕДНЕЕ ШЕСТИЛЕТИЕ (1899-1905), [Ехаmen des projets de réformes de l'école secondaire russe (1899-1905)]. ŽMNP, Partie 7 : 34-50, 73-122.

ТРУДЫ СОБРАНИЙ ПРЕПОДАВАТЕЛЕЙ ЯЗЫКОВ РУССКОГО И ИНОСТРАННЫХ. 1886-1887 УЧ. Г. (1888). [Travaux des enseignants des langues russe et étrangères. 1886-1887]. Saint-Pétersbourg : Musée pédagogique des établissements secondaires militaires.

UŠINSKIJ, Konstantin (1861). « РОдноЕ слово » [Langue natale]. ŽMNP, Partie 110 : 73-95.

VARON, Evgenij (1874). « О ПРЕПОДАВАНИИ ИНОСТРАННЫХ ЯЗЫКОВ ВООБЩЕ И ФРАНЦУЗСКОГО ЯзыкА в осоБЕнности », [De l'enseignement des langues étrangères en général, et du français, en particulier]. ŽMNP, Partie 175 : 58-95.

Outils didactiques : manuels, grammaires, chrestomathies, recueils de textes

CHALlANDES, Adamir (1902). Cours complet de langue francaise (1891-1902). Saint-Pétersbourg :

Trenké et Fusnot.

FABRICIEV, V. (1911) [1903]). ГРАММАТИКА ФРАНЦУЗСКОГО ЯЗЫКА СПЕЦИАЛЬНО ПРИСПОСОБЛЕННАЯ К УЧЕБНИКАМ, СОСТАВЛЕННЫМ ПО НАТУРАЛЬНОМУ МЕТОДУ, И ДЛЯ РАЗБОРА ФРАНЦУЗСКИХ ТЕКСТОВ, [Lе cours complet de grammaire française adapté à la méthode naturelle et à l'analyse de textes français]. Moscou : s.n.

FEUILLYE, Lucien \& MARTIN, Louis (1904). Nouveau cours de français d'après les principes de la méthode directe, naturelle ou intuitive, Saint-Pétersbourg : s.n.

FEUILLYE, Lucien \& MARTIN, Louis (1914). Morceaux choisis tirés des meilleurs auteurs français. Avec notices littéraires et vocabulaire. $14^{\mathrm{e}}$ éd. Saint-Pétersbourg : s.n.

Fenoult, Nicolas (1906). Premières leçons de français. Conversation, lecture et écriture à l'usage des familles et des écoles russes. $8^{\mathrm{e}}$ éd. Saint-Pétersbourg : Isakov.

FLEURY, Jean (1872). Histoire élémentaire de la littérature française. Saint-Pétersbourg et Moscou : s.n. FLEURY, Jean (1874). Extraits des meilleurs auteurs français, par ordre chronologique. Complément de l'Histoire abrégée de la littérature française. Saint-Pétersbourg : s.n.

IHNATOVICZ, Vitold (1899). Manuel concentrique de langue française comparée au russe et au latin divisé en trois parties. Cours élémentaire ( $3^{e}$ chapitre en texte suivi). $17^{\mathrm{e}}$ éd. Saint-Pétersbourg : D. D. Polubojarinov.

JACLARD-CORVIN, Victor (1878). Chrestomathie française pour les classes moyennes et les classes supérieures des établissements d'éducation, avec dictionnaire, introductions littéraires, notices biographiques, notes explicatives et exercices. T.1. Prose, T.2. Poésie. Saint-Pétersbourg : G. Ianpolski. KERCOFF, Aimé (1892). Cours gradué de langue française à l'usage des classes moyennes et supérieures des gymnases et des écoles militaires. Moscou : s.n. 
KLASSE, Octave (1908). Le petit français ou l'Enseignement par l'Image. Ouvrage ill. de 503 gravures. $7^{\mathrm{e}}$ éd. Vilno : Zavadski.

KonOF, Ekaterina (1904). Pour lire seul. Recueil de récits faciles. Série 1. Quand j'étais petit garçon. Moscou : Sotrudnik škol.

KONOF, Ekaterina (1914). Premier livre de lecture d'après la méthode naturelle. Causerie et lectures à l'usage des commençants. $16^{\mathrm{e}}$ éd. Moscou : Škola.

LANZEFF, E. (1909). Grammaire française. Théorie et pratique. Kazan : s.n.

MARTIN Louis (1904). Précis de grammaire française disposé par ordre alphabétique avec remarques sur quelques constructions et gallicismes. Approuvé par le Comité Scientifique du ministère de l'Instruction publique à l'usage des établissements secondaires. Saint-Pétersbourg : s.n.

MARTIN, Louis (1917-1918). Leçons de littérature française: Analyses et extraits des œuvres des meilleurs auteurs. (T. 1 : Des origines au XVIII ${ }^{e}$ siècle. T. 2. Du XVIII e siècle à nos jours). Nouv. éd., Petrograd : s.n. MARGOT, David (1905 [1855]). Cours élémentaire et progressif de la langue française à l'usage des classes inférieures et moyennes des écoles. $36^{\mathrm{e}}$ éd. Saint-Pétersbourg : s.n.

MARGOT, David (896) [1864]. Grammaire théorique et pratique de la langue française à l'usage des classes supérieures des écoles. $7^{\mathrm{e}}$ éd. Saint-Pétersbourg : s.n.

MIŽUEV, Pavel (1894). Chrestomathie française à l'usage des établissements secondaires. SaintPétersbourg : K .L. Rikker.

MIŽUEV, Pavel \& Olga (1911). Mosaïque française. Lectures littéraires tirées principalement des meilleurs auteurs du XIX ${ }^{e}$ siècle. $4^{\mathrm{e}}$ éd. Saint-Pétersbourg : K. L. Rikker.

MOSER, Pierre (1892). ФРАНЦУЗСКАЯ АЗБУКА. ЧТЕНИЕ И ПИСЬМО. РАЗГОВОР ПО НОВОМУ СПОСОБУ. СВЯЗНЫЕ СТАТЬИ С 33-МЯ РИСУНКАМИ. [L’Abécédaire français. Lecture et écriture. Conversation selon la nouvelle méthode. Textes suivis avec trente-trois dessins]. Moscou : Načalnaja škola.

MOSER, Pierre (1897). Cours pratique de langue française à l'usage des classes moyennes et supérieures de gymnases et progymnases. Par P. Moser, professeur de français au Corps des cadets sibérien. $5^{\mathrm{e}}$ éd. corrigée et augmentée ( 68 morceaux en prose et 12 en vers). Moscou : Lissner et Geschel.

PACHALERY, Anatole (1905). Anthologie des prosateurs et des poètes français du XIXe siècle. T.1 :

Prosateurs.(1800-1850). Odessa : G. Rousseau.

SILINA, Aleksandra (1895). Premières lectures et leçons de causerie par Al.S. $4^{\mathrm{e}}$ éd. revue et augmentée. Saint-Pétersbourg : Académie Impériale des sciences.

TRILLING, Philippe (1903). Cours pratique de langue française. T. 2. À la campagne et à la ville. SaintPétersbourg : Institut Lazarev.

VARON, Eugène (1878 [1874]). Cours élémentaire méthodique et pratique de langue française. Partie 2. Helsingfors : s.n.

Sources secondaires

CARRÈRE D’ENCAUSSE, Hélène (2008). Alexandre II, le printemps de la Russie. Paris : Fayard.

GANELIN, Šolom (1950). ОЧЕРКИ ПО ИСТОРИИ СРЕДНЕЙ ШКОЛЫ В РОССИИ [Essais d'une histoire de l'école secondaire russe]. Moscou-Léningrad : Učpedgiz.

KoRnILov, Aleksandre (1914). кУРС ИстоРИИ РОссии ХІХ ВЕКА, [Histoire de la Russie du XIX ${ }^{\mathrm{e}}$ siècle]. T.3. Moscou : M. et S. Sabašnikov. 
KouZMiNA, Nadejda (2005). « Les langues vivantes dans les établissements éducatifs russes au siècle des Lumières ». Documents pour l'histoire du français langue étrangère ou seconde, 35, 7-26.

KRIAJEVA, Nadejda (2017). «L'approche 'consciente et contrastive' de Fedor Buslaev, une innovation dans l'enseignement des langues en Russie du XIX ${ }^{\mathrm{e}}$ siècle ». Documents pour l'histoire du français langue étrangère ou seconde, 58/59, 61-81.

MIROLUBOV, Aleksandr (2002). ИСТОРИЯ ОТЕЧЕСТВЕННОЙ МЕТОДИКИ ОБУЧЕНИЯ ИНОСТРАННЫМ ЯзЫКАм, [Histoire de la didactique des langues étrangères en Russie]. Moscou : Stupeni-Infra.

RAXMANOV, Igor (réd.) (1972). ОСНОВНЫЕ НАПРАВЛЕНИЯ В МЕТОДИКЕ ПРЕПОДАВАНИЯ ИНОСТРАННЫХ языков в ХІХ-ХХ вв. [Didactiques des langues étrangères au XIX ${ }^{\mathrm{e}}$ et au XX $\mathrm{X}^{\mathrm{e}} \mathrm{s}$.], Moscou : Pedagogika.

RAUŠENBAX, Valentin (1971). КРАТКИЙ ОБЗОР ОСНОВНЫХ МЕТОДОВ ПРЕПОДАВАНИЯ ИНОСТРАННЫХ ЯзЫков С І По ХХ ВЕК [Abrégé de l'histoire des méthodes d'enseignement des langues étrangères $\mathrm{du} \mathrm{I}^{\mathrm{er}}$ au XX $\mathrm{X}^{\mathrm{e}}$ siècle]. Moscou : Vysšaja škola.

\section{NOTES}

1. Pourtant, ces écoles réales (РЕАЛЬНЫЕ учИлИщА) sont des établissements d'enseignement secondaire à part entière, à la différence des Realschulen allemandes. Les établissements secondaires de filles créés comme écoles de filles (1858) avec les deux LV obligatoires gardent le nom de gymnases qui leur a été attribué dès 1864 , le contenu de leurs programmes s'alignait sur celui des gymnases de garçons (avec pourtant plusieurs matières au volume horaire réduit).

2. Les écoles supérieures sont diverses: Institut des Mines à Saint-Pétersbourg (1836), École supérieure polytechnique à Moscou (1868), Instituts pédagogiques dans les villes universitaires. Les écoles militaires (corps des cadets) divisées entre 1862 et 1871 en gymnases militaires et écoles spécialisées supérieures reprennent leur dénomination initiale de corps des cadets tout en gardant la distinction en deux niveaux. Et c'est Tolstoï qui autorise l'ouverture des Cours supérieurs pour femmes (histoire et lettres, médecine, mathématiques, physique et chimie, pédagogie).

3. Curieusement les critiques émises par les historiens de la didactique des LV (Raušenbax 1971, Raxmanov 1972, Mirolubov 2002) ne se fondent que sur le cas des gymnases de garçons!

4. En témoigne l'actualité des travaux universitaires: Vetčinova Marina (2009). ТЕОРИЯ И ПРАКТИКА ИНОЯЗЫЧНОГО ОБРАЗОВАНИЯ В ОТЕЧЕСТВЕННОЙ ПЕДАГОГИКЕ ВТОРОЙ ПОЛОВИНЫ ХІХ - НАЧ. $X X$ в. [Théorisation et pratique de l'instruction par le biais des langues étrangères: seconde moitié du XIX ${ }^{\mathrm{e}}$ - début du XX $\mathrm{X}^{\mathrm{e}}$ s.], Thèse de doctorat. Université d'État de Koursk ; Nikšikova Lana (2007). ИСТОРИКО-ПЕДАГОГИЧЕСКИЕ ОСНОВЫ ПРЕПОДАВАНИЯ ИНОСТРАННЫХ ЯЗЫКОВ В РОССИИ ХІХ-НАЧ. $X X$ ВЕКА [Fondements historiques et pédagogiques de l'enseignement des langues étrangères dans la Russie du XIX $\mathrm{X}^{\mathrm{e}}$ - début du XX $\mathrm{e}$ s.] Thèse de doctorat. Université de Nižni Novgorod.

5. La Revue du ministère de l'Instruction publique [ЖУРНАЛ МИНИСТЕРСТВА НАРОДНОГО ПРОСВЕЩЕНИЯ] (1834-1917), par exemple, est mise entièrement en ligne: [http:// www.runivers.ru/lib/book7643/]; de même, les revues ВЕСтник ВоспитАния [Messager de l'éducation] (1890-1917), РУсСКАЯ ШКОЛА [École russe] (1890-1917), etc.

6. Par exemple, le fonds du Musée pédagogique des établissements secondaires militaires (1864-1918). Créé à Saint-Pétersbourg à l'initiative de leur directeur en chef Nikolaï Isakov (1821-1891), il réunissait et popularisait les collections d'outils didactiques de différents domaines et pays. La section des LV, l'une des plus actives, y œuvrait pour la promotion de la méthodologie directe en analysant les manuels de LV très nombreux à partir dès années 1890 . Cf. Barskov (1914) et тРуды соБРАнИй [Travaux des enseignants] (1888). 
7. La liste des ouvrages en LV à la fin de cet article résulte de recherches personnelles dans les bibliothèques russes.

8. УчЕБНЫЕ ПлАНЫ... [Plans et programmes d'études des gymnases...] (1890: 11).

9. David Margot (1823-1892), pédagogue d'origine suisse, enseigna le français à Saint-Pétersbourg dès 1846. Il fut directeur de l'école des paroisses réformées et lecteur de français à l'université de Saint-Pétersbourg (1858-1872). Il est l'auteur de manuels de français réédités jusqu'au début du $\mathrm{XX}^{\mathrm{e}}$ siècle.

10. De même, elle harmonisait l'usage de la terminologie grammaticale à l'école étant à l'origine de l'enseignement expérimental du russe et du latin par le même professeur dans les classes inférieures de gymnases.

11. Nicolas Ier s'opposa à l'introduction du grec ancien dans les plans d'études des gymnases en 1828, le traitant de «luxe », contrairement au français, " une sorte de nécessité » (Alešincev 1912 : 118).

12. Margot reconnaît pourtant à partir de la treizième édition (1872 [1855]) de son Cours élémentaire et progressif qu'il n'y a "rien de plus utile que les exercices de conversation », et que "dans l'étude des morceaux de la seconde partie, ces exercices deviennent un point essentiel »; la partie I étant intitulée depuis «Exercices de grammaire et de conversation 》 (Margot 1905 : VIIIIX).

13. УЧЕБНЫЕ ПЛАНЫ... ЖЕНСКИХ...[Plans d'études des LV dans les établissements secondaires de filles], (1874:150).

14. учЕБНЫЕ ПЛАНЫ... [Plans d'études (..) dans les écoles réales], (1879: 26).

15. Cf. aussi Dudyškin (1898:228-231).

16. Inspiré par les idées de la méthode « génétique » de Karl Mager, il en reconnaît pourtant les limites et mise sur l'avenir de la didactique des langues qui devrait créer une méthode optimale dans l'enseignement des LV.

17. Dans certains établissements on utilise parfois des ouvrages pour l'enseignement du français langue maternelle, comme par exemple: Frédéric Bataille Lectures enfantines (Paris 1894), ou encore adaptés à l'usage d'élèves russophones, par exemple une sélection d'histoires extraites du livre d'Émile Souvestre Au coin du feu (1857), avec introduction, commentaires et vocabulaire de Aleksandr Alekseev (1890).

\section{RÉSUMÉS}

La réforme du système de l'enseignement secondaire russe de 1871 ayant donné la priorité aux langues anciennes dans les gymnases de garçons, a également provoqué des modifications importantes dans l'enseignement des langues vivantes de ces établissements, mais aussi dans celui des écoles de type moderne (écoles réales, gymnases de filles, corps des cadets, etc.) C'est le contenu culturel (initiation à la lecture de chefs d'œuvre des littératures étrangères) qui va y être amplifié et mis au premier plan dans les deux types d'établissements se distinguant pourtant par le volume et les choix pédagogiques. La formation des compétences langagières de compréhension et d'expression orales et écrite, existant déjà auparavant dans les établissements de type moderne (en différentes proportions), sera renforcée par les progrès de la méthodologie directe et sa fusion réussie avec la méthodologie traditionnelle. 
The reform of the system of Russian secondary education of 1871 giving priority to ancient languages in boys' gymnasiums, has also brought about important changes in the teaching of the modern languages of these institutions, but also in the type schools modern (real schools, gymnasiums and girls' institutes, corps des cadets, etc.) It is the cultural content that will be amplified and put in the foreground in the two, yet distinguished by the volume and the educational choices. The training of oral and written language skills already existing in moderntype establishments will be considerably reinforced by the progress of the direct methodology and its successful fusion with the elements of the traditional.

\section{INDEX}

Mots-clés : école russe au XIXe siècle, langues vivantes, réforme de l'enseignement, diversification méthodologique

Keywords : Russian school in the 19th century, modern languages, educational reform, methodological diversification

\section{AUTEUR}

\section{NADÉJDA KRIAJEVA}

Université Clermont-Auvergne -nadejda.kriajeva@uca.fr 